\title{
Yagi-Uda Antenna Human Proximity Effects on the Link Budget at $300 \mathrm{MHz}$
}

\author{
Elyas Palantei ${ }^{(1)}$, and David V. Thiel *(2) \\ (1) Telecommunication \& Information Engineering, Electrical Engineering Department, \\ Faculty of Engineering, Hasanuddin University, Makassar, Indonesia 90245 \\ (1), (2) Centre for Wireless Monitoring \& Applications (CWMA) \\ School of Engineering, Griffith University - Nathan Q4111, Australia \\ E-mail: elyas_palantei@unhas.ac.id, e.palantei@griffith.edu.au, d.thiel@griffith.edu.au
}

\begin{abstract}
Yagi-Uda antennas are commonly used for data communications. Link budgets are calculated to ensure adequate fade margin. The presence of adjacent conducting material can affect antenna gain and impedance matching. Numerical modeling demonstrates that the gain of a 12 element Yagi-Uda is degraded by more than $2 \mathrm{~dB}$ when a human model is located in close proximity to the rear of the antenna. There are similar impedance matching losses. This has a significant effect on link reliability in RFID and wireless sensor networks where power is restricted.
\end{abstract}

Key words: Yagi-Uda antenna, human exposure, proximity effect, link budget.

\section{Introduction}

Yagi-Uda antennas are directional antennas used for such applications as radio location, data communications and TV receiving antennas, and they are usually employed in HF, VHF and UHF systems [1-2]. More recently these antennas are used in data transmission in sensor networks and RFID applications where the radiated power of the source is very limited.

Power link budget analysis is used to predict the quality and reliability of signal reception from a transmitter. The main purpose is to assess the link performance in terms of the bit error rate (BER) or the signal to noise ratio (SNR), at the far end reception link [3].

Many factors influence the communications link between transmitting and receiving antennas. For instance, Thiel [1] argued that the tower construction, the quality of antenna installation and human presence in the proximity of an installed antenna, affect the radiation properties. In particular applications, there are serious implications should the signal degrade significantly. Qualitatively, the loss of data is observed on a TV monitor when an airplane flies by. Ebersbach et al. [4] evaluated the human radiation exposure zone boundary distances close to a Yagi-Uda antenna. This paper reports further investigations on the effect of human presence close to this 12 element Yagi-Uda antenna [4].

\section{Numerical Experiments}

In this investigation, a numerical model was used to assess the impact on the gain and impedance of the antenna in free space, and with a human in close proximity. The 12 element Yagi-Uda antenna (one reflector, a driven element and 10 director elements) used in these investigations is described in [4]. The diameter of each wire element was 2 $\mathrm{cm}$. The antenna for [4] was re-sized and optimized to work at $300 \mathrm{MHz}$. The NEC program was run several times until an appropriate antenna model was obtained. The new model has the following elements dimensions: $L_{1}=0.402 \lambda_{0}, L_{2}=0.455 \lambda_{0}, L_{3}=0.3572 \lambda_{0}$, $L_{4}=0.335 \lambda_{0}, L_{5}=0.327 \lambda_{0}, L_{6}=0.318 \lambda_{0}, L_{7}=0.31 \lambda_{0}, L_{8}=0.31 \lambda_{0}, L_{9}=0.31 \lambda_{0}, L_{10}=0.31 \lambda_{0}$, $L_{11}=0.318 \lambda_{0}$, and $L_{12}=0.527 \lambda_{0}$. 
A human body was modeled in $3 \mathrm{D}$ as a set of connected wires with different orientation, radius and length. The model consisted of a head, two arms, torso, and two legs and each included resistive loads (see Fig. 1). The electrical properties of various human material (muscle, bone etc) varies with frequency [5]. The average conductivity $(\sigma)$ of the whole human body at $300 \mathrm{MHz}$ is approximately $1.477 \mathrm{~S} / \mathrm{m}$. The size of each human part in the model was: the head (including neck) was $20 \mathrm{~cm}$ (radius) and $25 \mathrm{~cm}$ (length); two arms, each of $10 \mathrm{~cm}$ (radius) and $50 \mathrm{~cm}$ (length); the torso was $30 \mathrm{~cm}$ (radius) and $50 \mathrm{~cm}$ (length); and two legs, each of $20 \mathrm{~cm}$ (radius) and $100 \mathrm{~cm}$ (length).

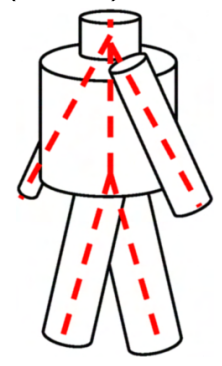

Figure 1: Six element NEC wire model of the human body.

\section{Results}

Figures 2 and 3 show the radiation patterns and the impedance mismatch (S11) for the antenna in free space, and the antenna with human $25 \mathrm{~cm}$ behind the director element. Fig.2a shows the radiation directional properties of the antenna in a free space (both elevation and azimuth). The principal lobe has a gain of approximately $13 \mathrm{dBi}$. The $50 \Omega$ impedance match is shown in Fig. 3. Results confirm some degradation of antenna performance due to the presence of the human. These effects result in reduction in main lobe gain of approximately 2 to $3 \mathrm{~dB}$ and a minor change in the S11.

The effect of the presence of a human working to install or maintain the antenna, an adjacent antenna or the tower itself, can affect link performance. This can be prevented if it is possible to restrict access to the area close to the antenna. The pattern variations due to the human presence in the proximity of the end of the reflector element of the YagiUda antenna are evident in Fig. 4. The change is more significant when a human moves in close proximity to the director elements. The gain variations in this situation are more than $5 \mathrm{~dB}$. This can extremely degrade the RF power level of communication link and decrease the read range significantly in RFID applications.

\section{Conclusions}

Depending on human traffic close to a Yagi-Uda antenna, there can be some degradation of the link. This must be taken into account when performance reliability measurements are quoted (estimated). In order to reduce effect of a human, the link design should include some additional antenna considerations such as:

- Increased antenna bandwidth

- Improved shielding in the vicinity of antenna

- Restricted human access inside $2 \lambda_{0}$ from the antenna extremities. This may not be possible in many situations.

\section{References:}

[1] D.V. Thiel, "Exposure zones near parasitic elements in high powered antennas", IEEE Antennas and Propagation Symposium, San Diego, CA, pp. 1-4, July 2008. 
[2] H.V. Cottony, "High gain antennas for VHF scatter propagation," IRE Transactions on Communication System, Vol.4, Issue 1, pp.56-63, March 1956.

[3] R.A. Santos, O. Alvarez, and A. Edwards, "Experimental analysis of wireless propagation model with mobile computing applications," $2^{\text {nd }}$ International Conference on Electrical and Electronics Engineering (ICEEE) and XI Conference on Electrical Engineering (CIE 2005), Mexico City, Mexico, Sept. 7-9, 2005: pp. 40-43.

[4] H. Ebersbach, D.V. Thiel and M. Leckenby, "Modelling Yagi-Uda antennas using point source approximation, " Electronics Letters, Vol. 40, (18), pp. 1095-1097, Sept. 2004.

[5] C.C. Johnson and A.W. Guy, "Nonionizing electromagnetic wave effects in biological materials and systems," Proc. IEEE, Vol. 60, pp. 692-718, June 1972.

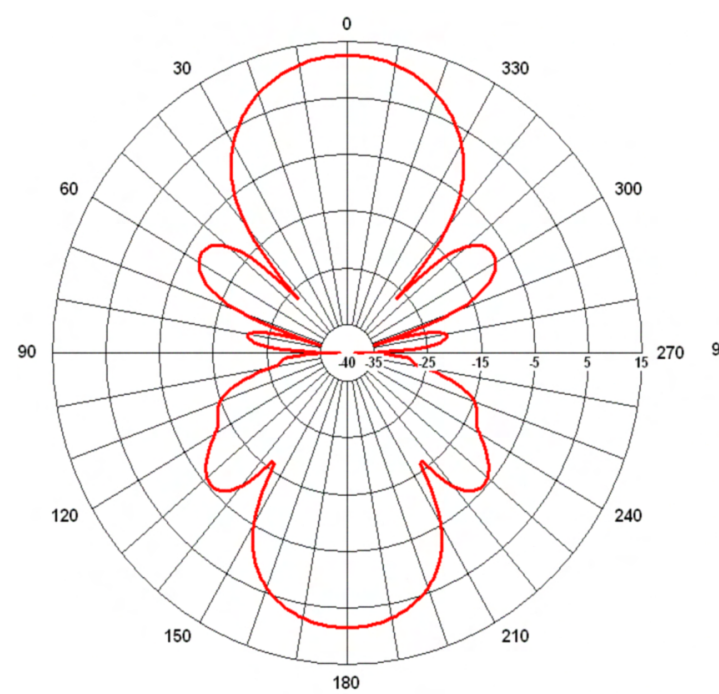

(a)

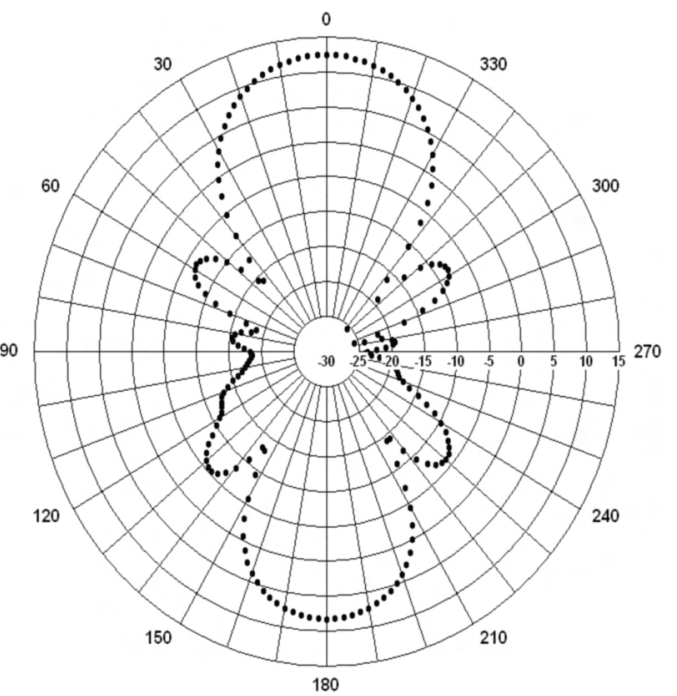

(b)

Figure 2: $\mathrm{H}$ plane radiation patterns of the Yagi-Uda antenna: (a) in free space (b) with human 25 $\mathrm{cm}$ behind the reflector parasitic element.

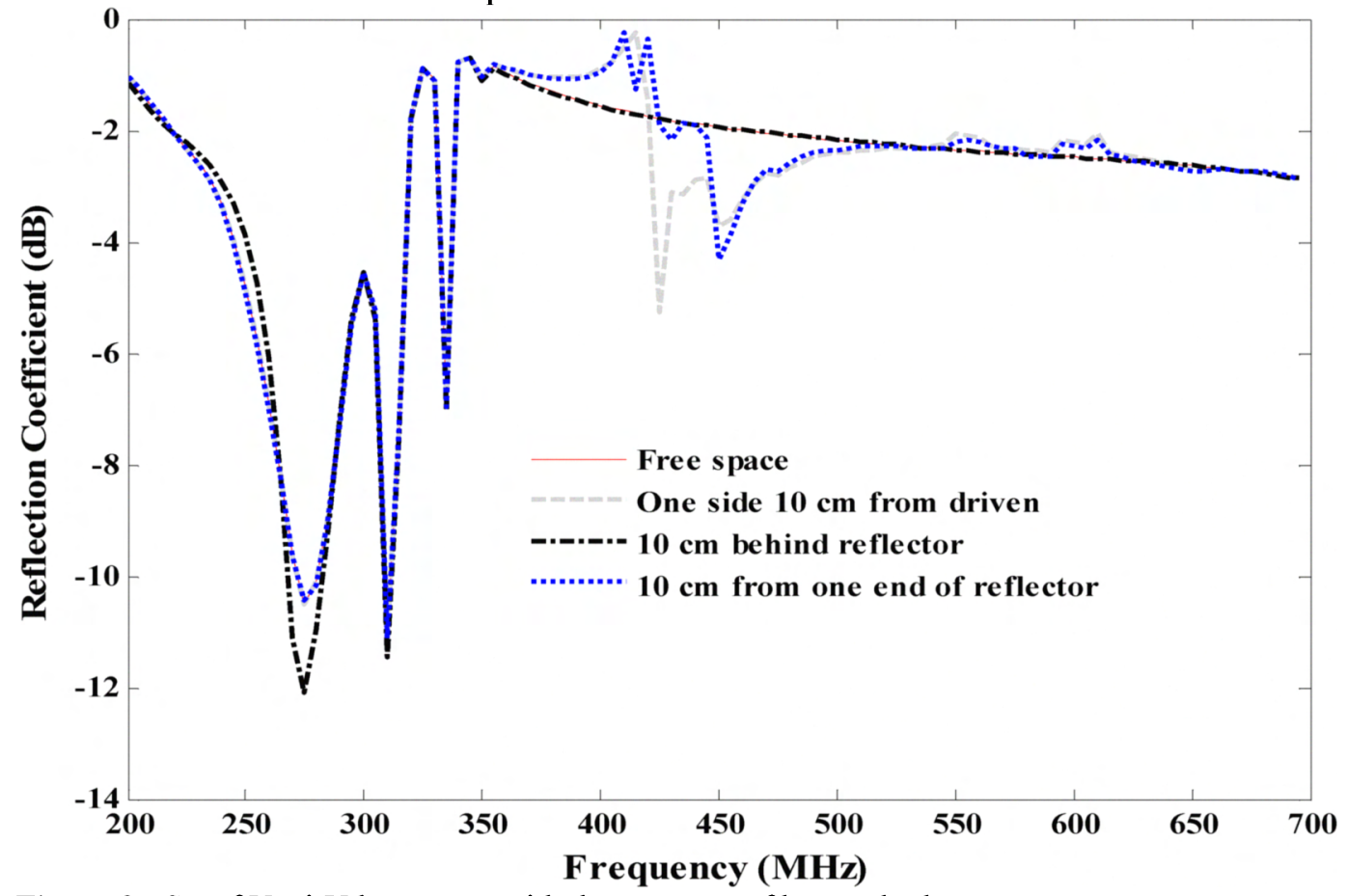

Figure 3: $S_{11}$ of Yagi-Uda antenna with the presence of human body. 


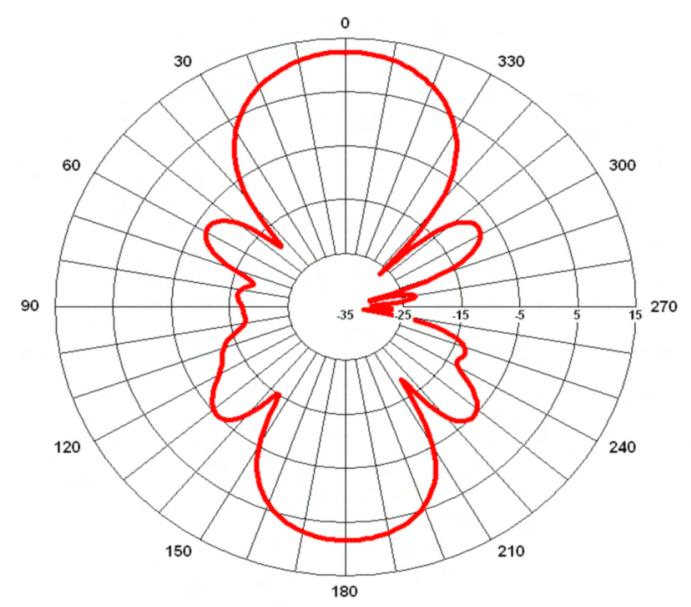

(i)

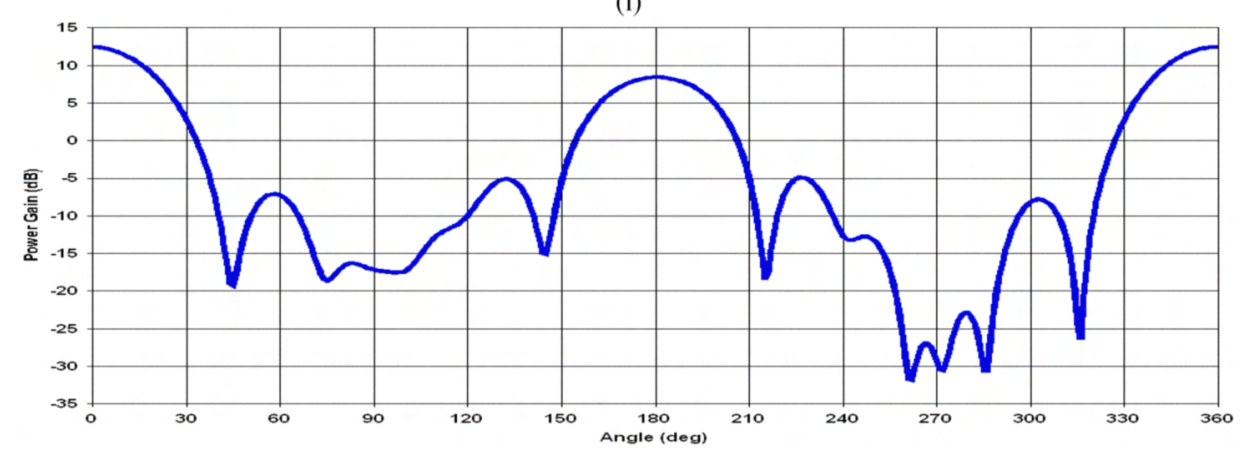

(ii)

(a). Azimuthal radiation pattern for a human model on the right side $10 \mathrm{~cm}$ of driven element: (i) polar, (ii) rectangular.

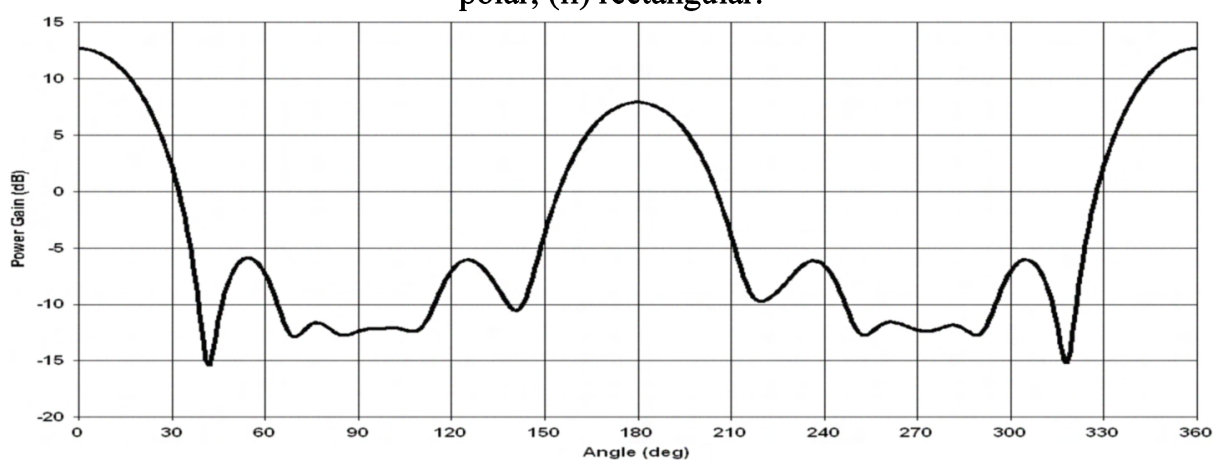

(b). Human model located $10 \mathrm{~cm}$ behind reflector element (at centre).

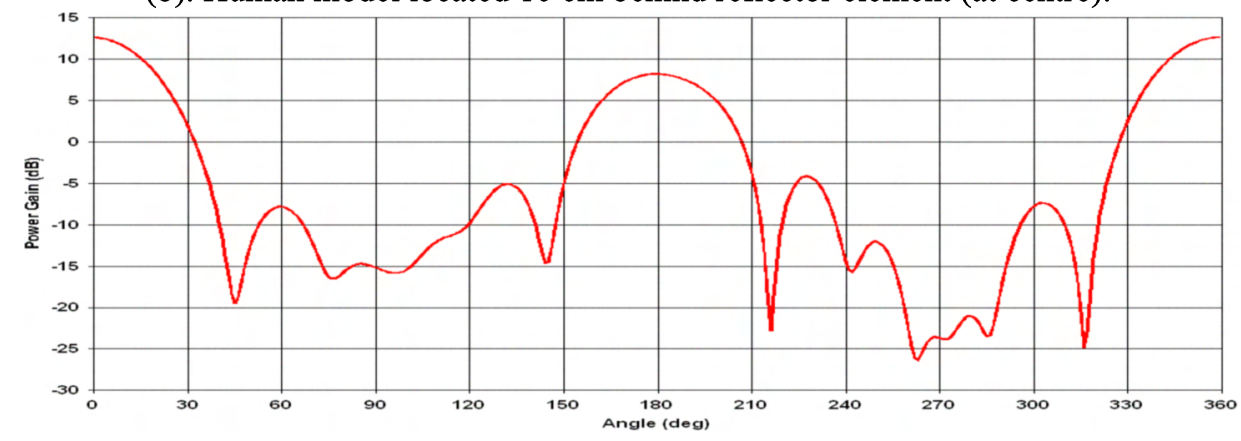

(c). Human model located $10 \mathrm{~cm}$ on the right end of the reflector element.

Figure 4: E plane radiation patterns as a function the position of the human model. 